\title{
Existence of nontrivial solutions for perturbed $p$-Laplacian system involving critical nonlinearity and magnetic fields
}

Huixing Zhang ${ }^{*}$, Jiaying Liu and Wenbin Liu

\section{"Correspondence:}

huixingzhangcumt@163.com Department of Mathematics, China

University of Mining and

Technology, Xuzhou, Jiangsu

221116, People's Republic of China

\begin{abstract}
Under the suitable assumptions, we establish the existence of nontrivial solutions for a perturbed $p$-Laplacian system in $\mathbb{R}^{N}$ with critical nonlinearity and magnetic fields by using the variational method.

MSC: 35B33; 35J60; 35J65
\end{abstract}

Keywords: $p$-Laplacian system; critical nonlinearity; magnetic fields; variational method

\section{Introduction}

In this paper, we consider a class of quasi-linear elliptic systems of the form

$$
\left\{\begin{array}{l}
-\varepsilon^{p} \Delta_{p, A} u+V(x)|u|^{p-2} u=H_{s}\left(|u|^{p},|v|^{p}\right)|u|^{p-2} u+K(x)|u|^{p^{*}-2} u, \quad x \in \mathbb{R}^{N}, \\
-\varepsilon^{p} \Delta_{p, A} v+V(x)|v|^{p-2} v=H_{t}\left(|u|^{p},|v|^{p}\right)|v|^{p-2} v+K(x)|v|^{p^{*}-2} v, \quad x \in \mathbb{R}^{N},
\end{array}\right.
$$

where $\Delta_{p, A} u=\operatorname{div}\left(|\nabla u+i A(x) u|^{p-2}(\nabla u+i A(x) u)\right), i$ is the imaginary unit, $A(x): \mathbb{R}^{N} \rightarrow \mathbb{R}^{N}$ is real vector potential, $1<p<N, V(x)$ is a non-negative potential, $p^{*}=N p /(N-p)$ denotes the Sobolev critical exponent for $N \geq 3$ and $K(x)$ is a bounded positive coefficient.

The scalar case corresponding to (1.1) has received considerable attention in recent years. For $p=2$ and $A(x) \equiv 0$, the scalar case corresponding to (1.1) turns into

$$
-\varepsilon^{2} \Delta u+V(x) u=K(x)|u|^{2^{*}-2} u+f\left(x,|u|^{2}\right) u, \quad x \in \mathbb{R}^{N} .
$$

The equation (1.2) arises in finding standing wave solutions of the nonlinear Schrödinger equation

$$
i \hbar \frac{\partial \psi}{\partial t}=-\frac{\hbar^{2}}{2 m} \Delta \psi+W(x) \psi-g(x,|\psi|) \psi
$$

A standing wave solution of (1.3) is a solution of the form

$$
\psi(x, t)=u(x) \exp ^{\frac{-i E t}{\hbar}} .
$$

Then $\psi(x, t)$ solves (1.3) if and only if $u(x)$ solves (1.2) with $V(x)=W(x)-E$ and $\varepsilon^{2}=\frac{\hbar^{2}}{2 m}$.

\section{Springer}

C 2013 Zhang et al.; licensee Springer. This is an Open Access article distributed under the terms of the Creative Commons Attribution License (http://creativecommons.org/licenses/by/2.0), which permits unrestricted use, distribution, and reproduction in any medium, provided the original work is properly cited. 
The equation (1.2) has been extensively investigated in the literature based on various assumptions of the potential $V(x)$ and the nonlinearity $f(x, u)$. See, for example, [1-15] and the references therein.

There are also many works dealing with the magnetic fields $A(x) \neq 0$ and $p=2$ for the scalar case corresponding to (1.1). In [16], the authors firstly obtained the existence of standing waves for special classes of magnetic fields. For many results, we refer the reader to $[17-22]$.

For general $p>1$, most of the work, as we know, consider the scalar case which corresponds to (1.1) with $A(x) \equiv 0$. See [23-27] and the references therein. We especially mention [24] for the existence of positive solutions for a class of $p$-Laplacian equations. Gloss [24] studied the existence and asymptotic behavior of positive solutions for quasi-linear elliptic equations of the form

$$
-\varepsilon^{p} \Delta_{p} u+V(x)|u|^{p-2} u=f(u), \quad x \in \mathbb{R}^{N},
$$

where $f$ is a subcritical nonlinearity without some growth conditions such as the Ambrosetti-Rabinowitz condition. The problem (1.4) has also been studied in [28-32]. The main difficulty in treating this class of equation (1.4) is a possible lack of compactness due to the unboundedness of the domain.

However, to our best knowledge, it seems there is almost no work on the existence of non-trivial solutions to the problem (1.1) involving critical nonlinearity and magnetic fields. We mainly follow the idea of [7]. Observe that though the idea was used in other problems, the adaption of the procedure to the problem is not trivial at all. Because of the appearance of magnetic fields $A(x)$, we must deal with the problem for complex-valued functions and therefore we need more delicate estimates.

The outline of the paper is as follows. The forthcoming section is the main result and preliminary results including the appropriate space setting to work with. In Section 3, we study the behavior of $(P S)_{c}$ sequence. Section 4 gets that the functional associated to the problem possesses the mountain geometry structure, and the last section concludes the proof of the main result.

\section{Main results and preliminaries}

Firstly, we make the following assumptions on $V(x), A(x), H(s, t)$ and $K(x)$ throughout the paper:

$\left(V_{0}\right) V \in C\left(\mathbb{R}^{N}, \mathbb{R}\right), V(0)=\inf _{x \in \mathbb{R}^{N}} V(x)=0$ and there exists $b>0$ such that the set $v^{b}:=$ $\left\{x \in \mathbb{R}^{N}: V(x)<b\right\}$ has finite Lebesgue measure;

$\left(A_{0}\right) A \in C\left(\mathbb{R}^{N}, \mathbb{R}^{N}\right)$ and $A(0)=0$;

$\left(K_{0}\right) K(x) \in C\left(\mathbb{R}^{N}, \mathbb{R}^{+}\right), 0<\inf K \leq \sup K<\infty$;

$\left(H_{1}\right) H(s, t) \in C^{1}\left(\mathbb{R}^{+} \times \mathbb{R}^{+}, \mathbb{R}\right)$ and $H_{s}, H_{t}=o(|s|+|t|)$ as $|s|+|t| \rightarrow 0$;

$\left(H_{2}\right)$ there exist $c_{1}>0$ and $p<\alpha<p^{*}$ such that

$$
\left|H_{s}(s, t)\right|,\left|H_{t}(s, t)\right| \leq c_{1}\left(1+|s|^{\frac{\alpha-p}{p}}+|t|^{\frac{\alpha-p}{p}}\right) ;
$$

$\left(H_{3}\right)$ there are $a_{0}>0, \theta \in\left(p, p^{*}\right)$ and $\alpha, \beta>p$ such that $H(s, t) \geq p a_{0}\left(|s|^{\frac{\alpha}{p}}+|t|^{\frac{\beta}{p}}\right)$ and $0<\frac{\theta}{p} H(s, t) \leq s H_{s}+t H_{t}$.

Under the above mentioned conditions, we get the following result. 
Theorem 1 Suppose that the assumptions $\left(V_{0}\right),\left(A_{0}\right),\left(K_{0}\right)$ and $\left(H_{1}\right)-\left(H_{3}\right)$ hold. Then for any $\sigma>0$, there is $\varepsilon_{\sigma}>0$ such that if $\varepsilon<\varepsilon_{\sigma}$, the problem (1.1) has at least one solution $\left(u_{\varepsilon}, v_{\varepsilon}\right)$ which satisfies

$$
\frac{\theta-p}{p \theta} \int_{\mathbb{R}^{N}}\left(\varepsilon^{p}\left(|\nabla| u_{\varepsilon}||^{p}+|\nabla| v_{\varepsilon}||^{p}\right)+V(x)\left|u_{\varepsilon}\right|^{p}+V(x)\left|v_{\varepsilon}\right|^{p}\right) \leq \sigma \varepsilon^{N} .
$$

Setting $\lambda=\varepsilon^{-p}$, the problem (1.1) is equivalent to the following problem:

$$
\left\{\begin{array}{l}
-\Delta_{p, A} u+\lambda V(x)|u|^{p-2} u=\lambda H_{s}\left(|u|^{p},|v|^{p}\right)|u|^{p-2} u+\lambda K(x)|u|^{p^{*}-2} u, \quad x \in \mathbb{R}^{N}, \\
-\Delta_{p, A} v+\lambda V(x)|v|^{p-2} v=\lambda H_{t}\left(|u|^{p},|v|^{p}\right)|v|^{p-2} v+\lambda K(x)|v|^{p^{*}-2} v, \quad x \in \mathbb{R}^{N} .
\end{array}\right.
$$

We are going to prove the following result.

Theorem 2 Suppose that the assumptions $\left(V_{0}\right),\left(A_{0}\right),\left(K_{0}\right)$ and $\left(H_{1}\right)-\left(H_{3}\right)$ hold. Then for any $\sigma>0$, there is $\Lambda_{\sigma}>0$ such that if $\lambda>\Lambda_{\sigma}$, the problem (2.1) has at least one solution $\left(u_{\lambda}, v_{\lambda}\right)$ which satisfies

$$
\frac{\theta-p}{p \theta} \int_{\mathbb{R}^{N}}\left(\left(|\nabla| u_{\lambda}||^{p}+|\nabla| v_{\lambda}||^{p}\right)+\lambda V(x)\left|u_{\lambda}\right|^{p}+\lambda V(x)\left|v_{\lambda}\right|^{p}\right) \leq \sigma \lambda^{1-\frac{p}{N}} .
$$

For convenience, we quote the following notations. Let $E_{\lambda, A}$ denote the Banach space

$$
E_{\lambda, A}=\left\{u \in W^{1, p}\left(\mathbb{R}^{N}\right): \int_{\mathbb{R}^{N}} \lambda V(x)|u|^{p}<\infty\right\}, \quad \lambda>0
$$

equipped with the norm

$$
\|u\|_{\lambda, A}=\left(\int_{\mathbb{R}^{N}}\left(\left|\nabla u+i \lambda^{\frac{1}{p}} A(x) u\right|^{p}+\lambda V(x)|u|^{p}\right)\right)^{\frac{1}{p}} .
$$

Set $E=E_{\lambda, A} \times E_{\lambda, A}$ and $\|(u, v)\|_{E}^{p}=\|u\|_{\lambda, A}^{p}+\|v\|_{\lambda, A}^{p}$ for any $(u, v) \in E$.

Similar to the diamagnetic inequality [16], we have

$$
|\nabla| u(x)|| \leq\left|\nabla u \frac{\bar{u}}{|u|}\right|=\left|\operatorname{Re}\left(\nabla u+i \lambda^{\frac{1}{p}} A u\right) \frac{\bar{u}}{|u|}\right| \leq\left|\nabla u+i \lambda^{\frac{1}{p}} A u\right|
$$

(the bar denotes complex conjugation). This inequality shows that if $u \in E_{\lambda, A}$, then $|u| \in$ $W^{1, p}\left(\mathbb{R}^{N}\right)$ and therefore $u \in L^{q}\left(\mathbb{R}^{N}\right)$ for any $q \in\left[p, p^{*}\right)$. That is to say, if $u_{n} \rightarrow u$ in $E_{\lambda, A}$, then $u_{n} \rightarrow u$ in $L_{\text {loc }}^{q}\left(\mathbb{R}^{N}\right)$ for any $q \in\left[p, p^{*}\right)$ and $u_{n} \rightarrow u$ a.e. in $\mathbb{R}^{N}$.

The energy functional associated with (2.1) is defined by

$$
\begin{aligned}
I_{\lambda}(u, v)= & \frac{1}{p} \int_{\mathbb{R}^{N}}\left(\left|\nabla u+i \lambda^{\frac{1}{p}} A u\right|^{p}+\lambda V(x)|u|^{p}+\left|\nabla v+i \lambda^{\frac{1}{p}} A v\right|^{p}+\lambda V(x)|v|^{p}\right) \\
& -\frac{\lambda}{p^{*}} \int_{\mathbb{R}^{N}} K(x)\left(|u|^{p^{*}}+|v|^{p^{*}}\right)-\frac{\lambda}{p} \int_{\mathbb{R}^{N}} H\left(|u|^{p},|v|^{p}\right) \\
= & \frac{1}{p}\|(u, v)\|_{E}^{p}-\lambda \int_{\mathbb{R}^{N}} G(u, v),
\end{aligned}
$$

where $G(u, v)=\frac{1}{p^{*}} K(x)\left(|u|^{p^{*}}+|v|^{p^{*}}\right)+\frac{1}{p} H\left(|u|^{p},|v|^{p}\right)$. 
Under the assumptions of Theorem 2, standard arguments [33] show that $I_{\lambda} \in C^{1}\left(E_{\lambda, A}\right.$, $\mathbb{R})$ and its critical points are weak solutions of the equation (2.1).

\section{$3(P S)_{c}$ condition}

We call a sequence $\left\{\left(u_{n}, v_{n}\right)\right\} \subset E$ a $(P S)_{c}$ sequence if $I_{\lambda}\left(u_{n}, v_{n}\right) \rightarrow c$ and $I_{\lambda}^{\prime}\left(u_{n}, v_{n}\right) \rightarrow 0$ strongly in $E^{\prime}$ ( $E^{\prime}$ is the dual space of $\left.E\right) . I_{\lambda}$ is said to satisfy the $(P S)_{c}$ condition if any $(P S)_{c}$ sequence contains a convergent subsequence.

The main result of Section 3 is the following compactness result.

Proposition 3.1 Let the assumptions of Theorem 2 be satisfied. There exists a constant $\alpha_{0} \geq 0$ independent of $\lambda$ such that, for any $(P S)_{c}$ sequence $\left\{\left(u_{n}, v_{n}\right)\right\} \subset E$ for $I_{\lambda}$ with $\left(u_{n}, v_{n}\right) \rightarrow(u, v)$, either $\left(u_{n}, v_{n}\right) \rightarrow(u, v)$ or $c-I_{\lambda}(u, v) \geq \alpha_{0} \lambda^{1-\frac{N}{p}}$.

As a consequence, we obtain the following result.

Proposition 3.2 Assume that the assumptions of Proposition 3.1 hold, $I_{\lambda}(u, v)$ satisfies the $(P S)_{c}$ condition for all $c \leq \alpha_{0} \lambda^{1-\frac{N}{p}}$.

In order to prove Proposition 3.1, we need the following lemmas.

Lemma 3.1 Let the assumptions of Theorem 2 be satisfied. $\left\{\left(u_{n}, v_{n}\right)\right\}$ is a $(P S)_{c}$ sequence of $I_{\lambda}$. Then $c \geq 0$ and $\left\{\left(u_{n}, v_{n}\right)\right\}$ is bounded in the space $E$.

Proof One has

$$
\begin{aligned}
& I_{\lambda}\left(u_{n}, v_{n}\right)-\frac{1}{\theta} I_{\lambda}^{\prime}\left(u_{n}, v_{n}\right)\left(u_{n}, v_{n}\right) \\
& =\left(\frac{1}{p}-\frac{1}{\theta}\right)\left\|\left(u_{n}, v_{n}\right)\right\|_{E}^{p}+\left(\frac{1}{\theta}-\frac{1}{p^{*}}\right) \lambda \int_{\mathbb{R}^{N}} K(x)\left(\left|u_{n}\right|^{p^{*}}+\left|v_{n}\right|^{p^{*}}\right) \\
& \quad+\lambda \int_{\mathbb{R}^{N}}\left(\frac{1}{\theta}\left(\left|u_{n}\right|^{p} H_{s}\left(\left|u_{n}\right|^{p},\left|v_{n}\right|^{p}\right)+\left|v_{n}\right|^{p} H_{t}\left(\left|u_{n}\right|^{p},\left|v_{n}\right|^{p}\right)\right)-\frac{1}{p} H\left(\left|u_{n}\right|^{p},\left|v_{n}\right|^{p}\right)\right) \\
& \geq\left(\frac{1}{p}-\frac{1}{\theta}\right)\left\|\left(u_{n}, v_{n}\right)\right\|_{E}^{p} .
\end{aligned}
$$

Together with $I_{\lambda}\left(u_{n}, v_{n}\right) \rightarrow c$ and $I_{\lambda}^{\prime}\left(u_{n}, v_{n}\right) \rightarrow 0$ as $n \rightarrow \infty$, we have

$$
\left(\frac{1}{p}-\frac{1}{\theta}\right)\left\|\left(u_{n}, v_{n}\right)\right\|_{E}^{p} \leq c+o(1)+\varepsilon_{n}\left\|\left(u_{n}, v_{n}\right)\right\|_{E} .
$$

Then $\left\{\left(u_{n}, v_{n}\right)\right\}$ is bounded and $c \geq 0$.

From Lemma 3.1, we may assume $\left(u_{n}, v_{n}\right) \rightarrow(u, v)$ in $E$ and $\left(u_{n}, v_{n}\right) \rightarrow(u, v)$ in $L_{\text {loc }}^{q}\left(\mathbb{R}^{N}\right) \times$ $L_{\text {loc }}^{q}\left(\mathbb{R}^{N}\right)$ for any $q \in\left[p, p^{*}\right)$ and $u_{n} \rightarrow u, v_{n} \rightarrow v$ a.e. in $\mathbb{R}^{N}$.

Lemma 3.2 Let $\gamma \in\left[p, p^{*}\right)$. There is a subsequence $\left\{\left(u_{n_{j}}, v_{n_{j}}\right)\right\}$ such that for any $\varepsilon>0$, there is $r_{\varepsilon}>0$ with $r \geq r_{\epsilon}$

$$
\lim _{j \rightarrow \infty} \sup \int_{B_{j} \backslash B_{r}}\left(\left|u_{n_{j}}\right|^{\gamma}+\left|v_{n_{j}}\right|^{\gamma}\right) \leq \varepsilon,
$$

where $B_{r}:=\left\{x \in \mathbb{R}^{N}:|x| \leq r\right\}$. 
Proof The proof of Lemma 3.2 is similar to that of Lemma 3.2 of [27], so we omit it.

Let $\eta \in C^{\infty}\left(\mathbb{R}^{+}\right)$be a smooth function satisfying $0 \leq \eta(t) \leq 1, \eta(t)=1$ if $t \leq 1$ and $\eta(t)=0$ if $t \geq 2$. Define $\widetilde{u}_{j}(x)=\eta(2|x| / j) u(x), \widetilde{v}_{j}(x)=\eta(2|x| / j) v(x)$. Obviously, we have

$$
\left\|u-\widetilde{u}_{j}\right\|_{E_{\lambda, A}} \rightarrow 0 \quad \text { and } \quad\left\|v-\widetilde{v}_{j}\right\|_{E_{\lambda, A}} \rightarrow 0 \quad \text { as } j \rightarrow \infty
$$

\section{Lemma 3.3 One has}

$$
\begin{aligned}
& \lim _{j \rightarrow \infty} \operatorname{Re} \int_{\mathbb{R}^{N}}\left(H_{s}\left(\left|u_{n_{j}}\right|^{p},\left|v_{n_{j}}\right|^{p}\right)\left|u_{n_{j}}\right|^{p-2} u_{n_{j}}\right. \\
& \left.\quad-H_{s}\left(\left|u_{n_{j}}-\widetilde{u}_{j}\right|^{p},\left|v_{n_{j}}-\widetilde{v}_{j}\right|^{p}\right)\left|u_{n_{j}}-\widetilde{u}_{j}\right|^{p-2}\left(u_{n_{j}}-\widetilde{u}_{j}\right)-H_{s}\left(\left|\widetilde{u}_{j}\right|^{p},\left|\widetilde{v}_{j}\right|^{p}\right)\left|\widetilde{u}_{j}\right|^{p-2} \widetilde{u}_{j}\right) \bar{\varphi}=0
\end{aligned}
$$

and

$$
\begin{aligned}
& \lim _{j \rightarrow \infty} \operatorname{Re} \int_{\mathbb{R}^{N}}\left(H_{t}\left(\left|u_{n_{j}}\right|^{p},\left|v_{n_{j}}\right|^{p}\right)\left|v_{n_{j}}\right|^{p-2} v_{n_{j}}\right. \\
& \left.\quad-H_{t}\left(\left|u_{n_{j}}-\widetilde{u}_{j}\right|^{p},\left|v_{n_{j}}-\widetilde{v}_{j}\right|^{p}\right)\left|v_{n_{j}}-\widetilde{v}_{j}\right|^{p-2}\left(v_{n_{j}}-\widetilde{v}_{j}\right)-H_{t}\left(\left|\widetilde{u}_{j}\right|^{p},\left|\widetilde{v}_{j}\right|^{p}\right)\left|\widetilde{v}_{j}\right|^{p-2} \widetilde{v}_{j}\right) \bar{\psi}=0
\end{aligned}
$$

uniformly in $(\varphi, \psi) \in E$ with $\|(\varphi, \psi)\|_{E} \leq 1$.

Proof The local compactness of Sobolev embedding implies that for any $r \geq 0$, we have

$$
\begin{aligned}
& \lim _{j \rightarrow \infty} \operatorname{Re} \int_{B_{r}}\left(H_{s}\left(\left|u_{n_{j}}\right|^{p},\left|v_{n_{j}}\right|^{p}\right)\left|u_{n_{j}}\right|^{p-2} u_{n_{j}}\right. \\
& \left.\quad-H_{s}\left(\left|u_{n_{j}}-\widetilde{u}_{j}\right|^{p},\left|v_{n_{j}}-\widetilde{v}_{j}\right|^{p}\right)\left|u_{n_{j}}-\widetilde{u}_{j}\right|^{p-2}\left(u_{n_{j}}-\widetilde{u}_{j}\right)-H_{s}\left(\left|\widetilde{u}_{j}\right|^{p},\left|\widetilde{v}_{j}\right|^{p}\right)\left|\widetilde{u}_{j}\right|^{p-2} \widetilde{u}_{j}\right) \bar{\varphi}=0
\end{aligned}
$$

uniformly in $\|\varphi\|_{E_{\lambda, A}} \leq 1$. For any $\varepsilon>0$, there exists $r_{\varepsilon}>0$ such that

$$
\lim _{j \rightarrow \infty} \sup \int_{B_{j} \backslash B_{r}}\left|\tilde{u}_{j}\right|^{\gamma} \leq \int_{\mathbb{R}^{N}}|u|^{\gamma} \leq \varepsilon
$$

for all $r \geq r_{\epsilon}$. Together with the assumption $\left(H_{2}\right)$ and the Hölder inequality, it follows from Lemma 3.2 that

$$
\begin{aligned}
\lim _{j \rightarrow \infty} & \sup \operatorname{Re} \int_{\mathbb{R}^{N}}\left(H_{s}\left(\left|u_{n_{j}}\right|^{p},\left|v_{n_{j}}\right|^{p}\right)\left|u_{n_{j}}\right|^{p-2} u_{n_{j}}\right. \\
& \left.-H_{s}\left(\left|u_{n_{j}}-\widetilde{u}_{j}\right|^{p},\left|v_{n_{j}}-\widetilde{v}_{j}\right|^{p}\right)\left|u_{n_{j}}-\widetilde{u}_{j}\right|^{p-2}\left(u_{n_{j}}-\widetilde{u}_{j}\right)-H_{s}\left(\left|\widetilde{u}_{j}\right|^{p},\left|\widetilde{v}_{j}\right|^{p}\right)\left|\widetilde{u}_{j}\right|^{p-2} \widetilde{u}_{j}\right) \bar{\varphi} \\
= & \lim _{j \rightarrow \infty} \sup \operatorname{Re} \int_{B_{j} \backslash B_{r}}\left(H_{s}\left(\left|u_{n_{j}}\right|^{p},\left|v_{n_{j}}\right|^{p}\right)\left|u_{n_{j}}\right|^{p-2} u_{n_{j}}\right. \\
& \left.\quad-H_{s}\left(\left|u_{n_{j}}-\widetilde{u}_{j}\right|^{p},\left|v_{n_{j}}-\widetilde{v}_{j}\right|^{p}\right)\left|u_{n_{j}}-\widetilde{u}_{j}\right|^{p-2}\left(u_{n_{j}}-\widetilde{u}_{j}\right)-H_{s}\left(\left|\widetilde{u}_{j}\right|^{p},\left|\widetilde{v}_{j}\right|^{p}\right)\left|\widetilde{u}_{j}\right|^{p-2} \widetilde{u}_{j}\right) \bar{\varphi} \\
\leq & c_{1} \lim _{j \rightarrow \infty} \sup \int_{B_{j} \backslash B_{r}}\left(\left|u_{n_{j}}\right|^{p-1}+\left|v_{n_{j}}\right|^{p-1}+\left|\widetilde{u}_{j}\right|^{p-1}+\left|\widetilde{v}_{j}\right|^{p-1}\right)|\bar{\varphi}| \\
& +c_{2} \lim _{j \rightarrow \infty} \sup \int_{B_{j} \backslash B_{r}}\left(\left|u_{n_{j}}\right|^{\alpha-1}+\left|v_{n_{j}}\right|^{\alpha-1}+\left|\widetilde{u}_{j}\right|^{\alpha-1}+\left|\widetilde{v}_{j}\right|^{\alpha-1}\right)|\bar{\varphi}|
\end{aligned}
$$




$$
\begin{aligned}
& \leq c_{1} \lim _{j \rightarrow \infty} \sup \left(\left\|u_{n_{j}}\right\|_{L^{p}\left(B_{j} \backslash B_{r}\right)}^{p-1}+\left\|v_{n_{j}}\right\|_{L^{p}\left(B_{j} \backslash B_{r}\right)}^{p-1}\right. \\
& \left.\quad+\left\|\widetilde{u}_{j}\right\|_{L^{p}\left(B_{j} \backslash B_{r}\right)}^{p-1}+\left\|\widetilde{v}_{j}\right\|_{L^{p}\left(B_{j} \backslash B_{r}\right)}^{p-1}\right)\|\bar{\varphi}\|_{L_{p}\left(B_{j} \backslash B_{r}\right)} \\
& \quad+c_{2} \lim _{j \rightarrow \infty} \sup \left(\left\|u_{n_{j}}\right\|_{L^{\alpha}\left(B_{j} \backslash B_{r}\right)}^{\alpha-1}+\left\|v_{n_{j}}\right\|_{L^{\alpha}\left(B_{j} \backslash B_{r}\right)}^{\alpha-1}\right. \\
& \left.\quad+\left\|\widetilde{u}_{j}\right\|_{L^{\alpha}\left(B_{j} \backslash B_{r}\right)}^{\alpha-1}+\left\|\widetilde{v}_{j}\right\|_{L^{\alpha}\left(B_{j} \backslash B_{r}\right)}^{\alpha-1}\right)\|\bar{\varphi}\|_{L_{\alpha}\left(B_{j} \backslash B_{r}\right)} \\
& \leq c_{3} \varepsilon^{\frac{p-1}{p}}+c_{4} \varepsilon^{\frac{\alpha-1}{\alpha}},
\end{aligned}
$$

where $c_{i}(i=1,2,3,4)$ are positive constants. Similarly, we can prove

$$
\begin{aligned}
& \lim _{j \rightarrow \infty} \operatorname{Re} \int_{\mathbb{R}^{N}}\left(H_{t}\left(\left|u_{n_{j}}\right|^{p},\left|v_{n_{j}}\right|^{p}\right)\left|v_{n_{j}}\right|^{p-2} v_{n_{j}}\right. \\
& \left.\quad-H_{t}\left(\left|u_{n_{j}}-\widetilde{u}_{j}\right|^{p},\left|v_{n_{j}}-\widetilde{v}_{j}\right|^{p}\right)\left|v_{n_{j}}-\widetilde{v}_{j}\right|^{p-2}\left(v_{n_{j}}-\widetilde{v}_{j}\right)-H_{t}\left(\left|\widetilde{u}_{j}\right|^{p},\left|\widetilde{v}_{j}\right|^{p}\right)\left|\widetilde{v}_{j}\right|^{p-2} \widetilde{v}_{j}\right) \bar{\psi}=0 .
\end{aligned}
$$

Lemma 3.4 Let $\left\{\left(u_{n}, v_{n}\right)\right\}$ and $\left\{\left(\widetilde{u}_{n}, \widetilde{v}_{n}\right)\right\}$ be as defined above. Then the following conclusions hold:

$$
I_{\lambda}\left(u_{n}-\widetilde{u}_{n}, v_{n}-\widetilde{v}_{n}\right) \rightarrow c-I_{\lambda}(u, v)
$$

and

$$
\left.I_{\lambda}^{\prime}\left(u_{n}-\widetilde{u}_{n}, v_{n}-\widetilde{v}_{n}\right) \rightarrow 0 \quad \text { in } E^{\prime} \text { (the dual space of } E\right)
$$

Proof By using the similar arguments of $[34,35]$, we have

$$
\begin{aligned}
I_{\lambda}\left(u_{n}\right. & \left.-\widetilde{u}_{n}, v_{n}-\widetilde{v}_{n}\right) \\
= & I_{\lambda}\left(u_{n}, v_{n}\right)-I_{\lambda}\left(\widetilde{u}_{n}, \widetilde{v}_{n}\right) \\
& +\frac{\lambda}{p^{*}} \int_{\mathbb{R}^{N}} K(x)\left(\left(\left|u_{n}\right|^{p^{*}}-\left|u_{n}-\widetilde{u}_{n}\right|^{p^{*}}-\left|\widetilde{u}_{n}\right|^{p^{*}}\right)+\left(\left|v_{n}\right|^{p^{*}}-\left|v_{n}-\widetilde{v}_{n}\right|^{p^{*}}-\left|\widetilde{v}_{n}\right|^{p^{*}}\right)\right) \\
& +\frac{\lambda}{p} \int_{\mathbb{R}^{N}}\left(H\left(\left|u_{n}\right|^{p},\left|v_{n}\right|^{p}\right)-H\left(\left|u_{n}-\widetilde{u}_{n}\right|^{p},\left|v_{n}-\widetilde{v}_{n}\right|^{p}\right)-H\left(\left|\widetilde{u}_{n}\right|^{p},\left|\widetilde{v}_{n}\right|^{p}\right)\right)+o(1) .
\end{aligned}
$$

By (3.1) and the similar idea of proving the Brézis-Lieb lemma [36], it is easy to get

$$
\lim _{n \rightarrow \infty} \int_{\mathbb{R}^{N}} K(x)\left(\left(\left|u_{n}\right|^{p^{*}}-\left|u_{n}-\widetilde{u}_{n}\right|^{p^{*}}-\left|\widetilde{u}_{n}\right|^{p^{*}}\right)+\left(\left|v_{n}\right|^{p^{*}}-\left|v_{n}-\widetilde{v}_{n}\right|^{p^{*}}-\left|\widetilde{v}_{n}\right|^{p^{*}}\right)\right)=0
$$

and

$$
\lim _{n \rightarrow \infty} \int_{\mathbb{R}^{N}}\left(H\left(\left|u_{n}\right|^{p},\left|v_{n}\right|^{p}\right)-H\left(\left|u_{n}-\widetilde{u}_{n}\right|^{p},\left|v_{n}-\widetilde{v}_{n}\right|^{p}\right)-H\left(\left|\widetilde{u}_{n}\right|^{p},\left|\widetilde{v}_{n}\right|^{p}\right)\right)=0
$$

Furthermore, using the fact $I_{\lambda}\left(u_{n}, v_{n}\right) \rightarrow c$ and $I_{\lambda}\left(\widetilde{u}_{n}, \widetilde{v}_{n}\right) \rightarrow I_{\lambda}(u, v)$, we obtain

$$
I_{\lambda}\left(u_{n}-\widetilde{u}_{n}, v_{n}-\widetilde{v}_{n}\right) \rightarrow c-I_{\lambda}(u, v) .
$$


In order to prove $I_{\lambda}^{\prime}\left(u_{n}-\widetilde{u}_{n}, v_{n}-\widetilde{v}_{n}\right) \rightarrow 0$ in $E^{-1}$, for any $(\varphi, \psi) \in E$, it follows that

$$
\begin{aligned}
I_{\lambda}^{\prime}\left(u_{n}\right. & \left.-\widetilde{u}_{n}, v_{n}-\widetilde{v}_{n}\right)(\varphi, \psi) \\
= & I_{\lambda}^{\prime}\left(u_{n}, v_{n}\right)(\varphi, \psi)-I_{\lambda}^{\prime}\left(\widetilde{u}_{n}, \widetilde{v}_{n}\right)(\varphi, \psi) \\
& +\lambda \operatorname{Re} \int_{\mathbb{R}^{N}} K(x)\left(\left|u_{n}\right|^{p^{*}-2} u_{n}-\left|u_{n}-\widetilde{u}_{n}\right|^{p^{*}-2}\left(u_{n}-\widetilde{u}_{n}\right)-\left|\widetilde{u}_{n}\right|^{p^{*}-2} \widetilde{u}_{n}\right) \bar{\varphi} \\
& +\lambda \operatorname{Re} \int_{\mathbb{R}^{N}} K(x)\left(\left|v_{n}\right|^{p^{*}-2} v_{n}-\left|v_{n}-\widetilde{v}_{n}\right|^{p^{*}-2}\left(v_{n}-\widetilde{v}_{n}\right)-\left|\widetilde{v}_{n}\right|^{p^{*}-2} \widetilde{v}_{n}\right) \bar{\psi} \\
& +\lambda \operatorname{Re} \int_{\mathbb{R}^{N}}\left(H_{s}\left(\left|u_{n}\right|^{p},\left|v_{n}\right|^{p}\right)\left|u_{n}\right|^{p-2} u_{n}\right. \\
& \left.-H_{s}\left(\left|u_{n}-\widetilde{u}_{n}\right|^{p},\left|v_{n}-\widetilde{v}_{n}\right|^{p}\right)\left|u_{n}-\widetilde{u}_{n}\right|^{p-2}\left(u_{n}-\widetilde{u}_{n}\right)-H_{s}\left(\left|\widetilde{u}_{n}\right|^{p},\left|\widetilde{v}_{n}\right|^{p}\right)\left|\widetilde{u}_{n}\right|^{p-2} \widetilde{u}_{n}\right) \bar{\varphi} \\
& +\lambda \operatorname{Re} \int_{\mathbb{R}^{N}}\left(H_{t}\left(\left|u_{n}\right|^{p},\left|v_{n}\right|^{p}\right)\left|v_{n}\right|^{p-2} v_{n}\right. \\
& \left.-H_{t}\left(\left|u_{n}-\widetilde{u}_{n}\right|^{p},\left|v_{n}-\widetilde{v}_{n}\right|^{p}\right)\left|v_{n}-\widetilde{v}_{n}\right|^{p-2}\left(v_{n}-\widetilde{v}_{n}\right)-H_{t}\left(\left|\widetilde{u}_{n}\right|^{p},\left|\widetilde{v}_{n}\right|^{p}\right)\left|\widetilde{v}_{n}\right|^{p-2} \widetilde{v}_{n}\right) \bar{\psi} \\
& +o(1) .
\end{aligned}
$$

It is standard to check that

$$
\lim _{n \rightarrow \infty} \int_{\mathbb{R}^{N}} K(x)\left(\left|u_{n}\right|^{p^{*}-2} u_{n}-\left|u_{n}-\widetilde{u}_{n}\right|^{p^{*}-2}\left(u_{n}-\widetilde{u}_{n}\right)-\left|\widetilde{u}_{n}\right|^{p^{*}-2} \widetilde{u}_{n}\right) \bar{\varphi}=0
$$

and

$$
\lim _{n \rightarrow \infty} \int_{\mathbb{R}^{N}} K(x)\left(\left|v_{n}\right|^{p^{*}-2} v_{n}-\left|v_{n}-\widetilde{v}_{n}\right|^{p^{*}-2}\left(v_{n}-\widetilde{v}_{n}\right)-\left|\widetilde{v}_{n}\right|^{p^{*}-2} \widetilde{v}_{n}\right) \bar{\psi}=0
$$

uniformly in $(\varphi, \psi) \in E$ with $\|(\varphi, \psi)\|_{E} \leq 1$. Together with Lemma 3.3, we have

$$
I_{\lambda}^{\prime}\left(u_{n}-\widetilde{u}_{n}, v_{n}-\widetilde{v}_{n}\right) \rightarrow 0 \quad \text { in } E^{\prime} .
$$

Let $u_{n}^{1}=u_{n}-\widetilde{u}_{n}, v_{n}^{1}=v_{n}-\widetilde{v}_{n}$, then $u_{n}-u=u_{n}^{1}+\left(\widetilde{u}_{n}-u\right), v_{n}-v=v_{n}^{1}+\left(\widetilde{v}_{n}-v\right)$. From (3.1), we get $\left(u_{n}, v_{n}\right) \rightarrow(u, v)$ in $E$ if and only if $\left(u_{n}^{1}, v_{n}^{1}\right) \rightarrow(0,0)$ in $E$.

Observe that

$$
\begin{aligned}
I_{\lambda}\left(u_{n}^{1}, v_{n}^{1}\right)-\frac{1}{p} I_{\lambda}^{\prime}\left(u_{n}^{1}, v_{n}^{1}\right)\left(u_{n}^{1}, v_{n}^{1}\right) \\
=\left(\frac{1}{p}-\frac{1}{p^{*}}\right) \lambda \int_{\mathbb{R}^{N}} K(x)\left(\left|u_{n}^{1}\right|^{p^{*}}+\left|v_{n}^{1}\right|^{p^{*}}\right) \\
\quad+\frac{\lambda}{p} \int_{\mathbb{R}^{N}}\left(\left|u_{n}^{1}\right|^{p} H_{s}\left(\left|u_{n}^{1}\right|^{p},\left|v_{n}^{1}\right|^{p}\right)+\left|v_{n}^{1}\right|^{p} H_{t}\left(\left|u_{n}^{1}\right|^{p},\left|v_{n}^{1}\right|^{p}\right)-H\left(\left|u_{n}^{1}\right|^{p},\left|v_{n}^{1}\right|^{p}\right)\right) \\
\geq \frac{\lambda}{N} \int_{\mathbb{R}^{N}} K(x)\left(\left|u_{n}^{1}\right|^{p^{*}}+\left|v_{n}^{1}\right|^{p^{*}}\right) \\
\geq \frac{\lambda}{N} K_{\min } \int_{\mathbb{R}^{N}}\left(\left|u_{n}^{1}\right|^{p^{*}}+\left|v_{n}^{1}\right|^{p^{*}}\right),
\end{aligned}
$$

where $K_{\min }=\inf _{x \in \mathbb{R}^{N}} K(x)>0$. Furthermore, we get

$$
\left\|\left(u_{n}^{1}, v_{n}^{1}\right)\right\|_{p^{*}}^{p^{*}} \leq \frac{N\left(c-I_{\lambda}(u, v)\right)}{\lambda K_{\min }}+o(1) .
$$


Now, we consider the energy level of the functional $I_{\lambda}$ below which the $(P S)_{c}$ condition holds.

Let $V_{b}(x):=\max \{V(x), b\}$, where $b$ is a positive constant in the assumption $\left(V_{0}\right)$. Since the set $v_{b}$ has finite measure, we get

$$
\int_{\mathbb{R}^{N}} V(x)\left(\left|u_{n}^{1}\right|^{p}+\left|v_{n}^{1}\right|^{p}\right)=\int_{\mathbb{R}^{N}} V_{b}(x)\left(\left|u_{n}^{1}\right|^{p}+\left|v_{n}^{1}\right|^{p}\right)+o(1) .
$$

In connection with the assumptions $\left(H_{1}\right)-\left(H_{3}\right)$ and the Young inequality, there exists $C_{b}>0$ such that

$$
\begin{aligned}
& \int_{\mathbb{R}^{N}}\left(K(x)\left(|u|^{p^{*}}+|v|^{p^{*}}\right)+|u|^{p} H_{s}\left(|u|^{p},|v|^{p}\right)+|v|^{p} H_{t}\left(|u|^{p},|v|^{p}\right)\right) \\
& \quad \leq b\left(\|u\|_{p}^{p}+\|v\|_{p}^{p}\right)+C_{b}\left(\|u\|_{p^{*}}^{p^{*}}+\|v\|_{p^{*}}^{p^{*}}\right) .
\end{aligned}
$$

Let $S$ be the best Sobolev constant of the immersion

$$
S\|u\|_{p^{*}}^{p} \leq \int_{\mathbb{R}^{N}}|\nabla u|^{p} \quad \text { for all } u \in W^{1, p}\left(\mathbb{R}^{N}\right) .
$$

Proof of Proposition 3.1 Assume that $\left(u_{n}, v_{n}\right) \nrightarrow(u, v)$, then

$$
\lim \inf _{n \rightarrow \infty}\left\|\left(u_{n}^{1}, v_{n}^{1}\right)\right\|_{E}>0
$$

and

$$
c-I_{\lambda}(u, v)>0 .
$$

By the Sobolev embedding inequality and the diamagnetic inequality, we get

$$
\begin{aligned}
S( & \left.\left\|u_{n}^{1}\right\|_{p^{*}}^{p}+\left\|v_{n}^{1}\right\|_{p^{*}}^{p}\right) \\
\leq & \int_{\mathbb{R}^{N}}\left(\left|\nabla u_{n}^{1}\right|^{p}+\left|\nabla v_{n}^{1}\right|^{p}\right) \\
\leq & \int_{\mathbb{R}^{N}}\left(\left|\nabla u_{n}^{1}+i \lambda^{\frac{1}{p}} A(x) u_{n}^{1}\right|^{p}+\left|\nabla v_{n}^{1}+i \lambda^{\frac{1}{p}} A(x) v_{n}^{1}\right|^{p}\right) \\
= & \int_{\mathbb{R}^{N}}\left(\left|\nabla u_{n}^{1}+i \lambda^{\frac{1}{p}} A(x) u_{n}^{1}\right|^{p}+\lambda V(x)\left|u_{n}^{1}\right|^{p}+\left|\nabla v_{n}^{1}+i \lambda^{\frac{1}{p}} A(x) v_{n}^{1}\right|^{p}+\lambda V(x)\left|v_{n}^{1}\right|^{p}\right) \\
& -\lambda \int_{\mathbb{R}^{N}} V(x)\left(\left|u_{n}^{1}\right|^{p}+\left|v_{n}^{1}\right|^{p}\right) \\
= & \lambda \int_{\mathbb{R}^{N}} K(x)\left(\left|u_{n}^{1}\right|^{p^{*}}+\left|v_{n}^{1}\right|^{p^{*}}\right)+\left|u_{n}^{1}\right|^{p} H_{s}\left(\left|u_{n}^{1}\right|^{p},\left|v_{n}^{1}\right|^{p}\right)+\left|v_{n}^{1}\right|^{p} H_{t}\left(\left|u_{n}^{1}\right|^{p},\left|v_{n}^{1}\right|^{p}\right) \\
& -\lambda \int_{\mathbb{R}^{N}} V_{b}(x)\left(\left|u_{n}^{1}\right|^{p}+\left|v_{n}^{1}\right|^{p}\right)+o(1) \\
\leq & \lambda b\left(\left\|u_{n}^{1}\right\|_{p}^{p}+\left\|v_{n}^{1}\right\|_{p}^{p}\right)+\lambda C_{b}\left(\left\|u_{n}^{1}\right\|_{p^{*}}^{p^{*}}+\left\|v_{n}^{1}\right\|_{p^{*}}^{p^{*}}\right)-\lambda b\left(\left\|u_{n}^{1}\right\|_{p}^{p}+\left\|v_{n}^{1}\right\|_{p}^{p}\right)+o(1) \\
= & \lambda C_{b}\left(\left\|u_{n}^{1}\right\|_{p^{*}}^{p^{*}}+\left\|v_{n}^{1}\right\|_{p^{*}}^{p^{*}}\right)+o(1) .
\end{aligned}
$$


This, together with (3.2), gives

$$
\begin{aligned}
S & \leq \lambda C_{b}\left(\left\|u_{n}^{1}\right\|_{p^{*}}^{p^{*}}+\left\|v_{n}^{1}\right\|_{p^{*}}^{p^{*}}\right)^{\frac{p^{*}-p}{p^{*}}}+o(1) \\
& \leq \lambda C_{b}\left(\frac{N\left(c-I_{\lambda}(u, v)\right)}{\lambda K_{\min }}\right)^{\frac{p}{N}}+o(1) \\
& =\lambda^{1-\frac{p}{N}} C_{b}\left(\frac{N}{K_{\min }}\right)^{\frac{p}{N}}\left(c-I_{\lambda}(u, v)\right)^{\frac{p}{N}}+o(1) .
\end{aligned}
$$

Set $\alpha_{0}=S^{\frac{N}{p}} C_{b}^{-\frac{N}{p}} N^{-1} K_{\min }$, then

$$
\alpha_{0} \lambda^{1-\frac{N}{p}} \leq c-I_{\lambda}(u, v)+o(1)
$$

This completes the proof of Proposition 3.1.

Proof of Proposition 3.2 Since $c \leq \alpha_{0} \lambda^{1-\frac{N}{p}}$, we have

$$
c-I_{\lambda}(u, v) \leq \alpha_{0} \lambda^{1-\frac{N}{p}}-I_{\lambda}(u, v) .
$$

In connection with $I_{\lambda}(u, v) \geq 0$ and Proposition 3.1, we complete this proof.

\section{The mountain-pass structure}

In the following, we always consider $\lambda \geq 1$. We will prove that $I_{\lambda}$ possesses the mountainpass structure which has been carefully discussed in the works [37, 38].

Lemma 4.1 Let the assumptions of Theorem 2 be satisfied. There exist $\alpha_{\lambda}, \rho_{\lambda}>0$ such that

$$
I_{\lambda}(u, v)>0 \quad \text { if } 0<\|(u, v)\|_{E}<\rho_{\lambda} \quad \text { and } \quad I_{\lambda}(u, v) \geq \alpha_{\lambda} \quad \text { if }\|(u, v)\|_{E}=\rho_{\lambda} .
$$

Proof By (3.4), for any $\delta>0$, there is $C_{\delta}>0$ such that

$$
\int_{\mathbb{R}^{N}} G(u, v) \leq \delta\left(\|u\|_{p}^{p}+\|v\|_{p}^{p}\right)+C_{\delta}\left(\|u\|_{p^{*}}^{p^{*}}+\|v\|_{p^{*}}^{p^{*}}\right) .
$$

Thus,

$$
\begin{aligned}
I_{\lambda}(u, v) & =\frac{1}{p}\|(u, v)\|_{E}^{p}-\lambda \int_{\mathbb{R}^{N}} G(u, v) \\
& \geq \frac{1}{p}\|(u, v)\|_{E}^{p}-\lambda \delta\left(\|u\|_{p}^{p}+\|v\|_{p}^{p}\right)-\lambda C_{\delta}\left(\|u\|_{p^{*}}^{p^{*}}+\|v\|_{p^{*}}^{p^{*}}\right) .
\end{aligned}
$$

In connection with $\|u\|_{p}^{p}+\|v\|_{p}^{p} \leq C_{1}\|(u, v)\|_{E}^{p}$, we may choose $\delta \leq\left(2 p \lambda C_{1}\right)^{-1}$ such that

$$
I_{\lambda}(u, v) \geq \frac{1}{2 p}\|(u, v)\|_{E}^{p}-\lambda C_{\delta}\left(\|u\|_{p^{*}}^{p^{*}}+\|v\|_{p^{*}}^{p^{*}}\right) .
$$

The fact $p^{*}>p$ implies the desired conclusion. 
Lemma 4.2 Under the assumptions of Lemma 4.1, for any finite dimensional subspace $F \subset E$, we have

$$
I_{\lambda}(u, v) \rightarrow-\infty \quad \text { as }(u, v) \in F, \quad\|(u, v)\|_{E} \rightarrow \infty .
$$

Proof Together with the fact $H(s, t) \geq p a_{0}\left(|s|^{\frac{\alpha}{p}}+|t|^{\frac{\beta}{p}}\right)$, we have

$$
I_{\lambda}(u, v) \leq \frac{1}{p}\|(u, v)\|_{E}^{p}-\lambda a_{0}\left(\|u\|_{\alpha}^{\alpha}+\|v\|_{\beta}^{\beta}\right) \quad \text { for all }(u, v) \in E .
$$

Since all norms in a finite-dimensional space are equivalent and $\alpha, \beta>p$, we complete the proof.

In the following, we will find special finite-dimensional subspaces by which we establish sufficiently small mini-max levels.

Define the functional

$$
\Phi_{\lambda}(u, v)=\frac{1}{p}\|(u, v)\|_{E}^{p}-\lambda a_{0} \int_{\mathbb{R}^{N}}\left(|u|^{\alpha}+|v|^{\beta}\right) .
$$

Obviously, it follows that $\Phi_{\lambda} \in C^{1}(E)$ and $I_{\lambda}(u, v) \leq \Phi_{\lambda}(u, v)$ for all $(u, v) \in E$.

Observe that

$$
\inf \left\{\int_{\mathbb{R}^{N}}|\nabla \phi|^{p}: \phi \in C_{0}^{\infty}\left(\mathbb{R}^{N}, \mathbb{R}\right),\|\phi\|_{L^{\alpha}\left(\mathbb{R}^{N}\right)}=1\right\}=0
$$

and

$$
\inf \left\{\int_{\mathbb{R}^{N}}|\nabla \psi|^{p}: \psi \in C_{0}^{\infty}\left(\mathbb{R}^{N}, \mathbb{R}\right),\|\psi\|_{L^{\beta}\left(\mathbb{R}^{N}\right)}=1\right\}=0 .
$$

Then, for any $\delta>0$, there are $\phi_{\delta}, \psi_{\delta} \in C_{0}^{\infty}\left(\mathbb{R}^{N}, \mathbb{R}\right)$ with $\left\|\phi_{\delta}\right\|_{L^{\alpha}\left(\mathbb{R}^{N}\right)}=\left\|\psi_{\delta}\right\|_{L^{\beta}\left(\mathbb{R}^{N}\right)}=1$ and $\operatorname{supp} \phi_{\delta}$, supp $\psi_{\delta} \subset B_{r_{\delta}}(0)$ such that $\left\|\nabla \phi_{\delta}\right\|_{p}^{p},\left\|\nabla \psi_{\delta}\right\|_{p}^{p}<\delta$.

Set $e_{\lambda}(x)=\left(\phi_{\delta}(\sqrt[p]{\lambda} x), \psi_{\delta}(\sqrt[p]{\lambda} x)\right)$. Then supp $e_{\lambda} \subset B_{\lambda^{-\frac{1}{p}} r_{\delta}}(0)$. For $t \geq 0$, we get

$$
\begin{aligned}
\Phi_{\lambda}\left(t e_{\lambda}\right) & =\frac{t^{p}}{p}\left\|e_{\lambda}\right\|_{E}^{p}-a_{0} \lambda t^{\alpha} \int_{\mathbb{R}^{N}}\left|\phi_{\delta}(\sqrt[p]{\lambda} x)\right|^{\alpha}-a_{0} \lambda t^{\beta} \int_{\mathbb{R}^{N}}\left|\psi_{\delta}(\sqrt[p]{\lambda} x)\right|^{\beta} \\
& =\lambda^{1-\frac{N}{p}} J_{\lambda}\left(t \phi_{\delta}, t \psi_{\delta}\right),
\end{aligned}
$$

where

$$
\begin{aligned}
J_{\lambda}(u, v)= & \frac{1}{p} \int_{\mathbb{R}^{N}}\left(|\nabla u|^{p}+|\nabla v|^{p}+\left(A\left(\lambda^{-\frac{1}{p}} x\right)+V\left(\lambda^{-\frac{1}{p}} x\right)\right)\left(|u|^{p}+|v|^{p}\right)\right) \\
& -a_{0} \int_{\mathbb{R}^{N}}\left(|u|^{\alpha}+|v|^{\beta}\right) .
\end{aligned}
$$

By direct computation, we have

$$
\max _{t \geq 0} J_{\lambda}\left(t \phi_{\delta}, t \psi_{\delta}\right) \leq \frac{\alpha-p}{p \alpha\left(\alpha a_{0}\right)^{\frac{p}{\alpha-p}}}\left\{\int_{\mathbb{R}^{N}}\left(\left|\nabla \phi_{\delta}\right|^{p}+\left(A\left(\lambda^{-\frac{1}{p}} x\right)+V\left(\lambda^{-\frac{1}{p}} x\right)\right)\left|\phi_{\delta}\right|^{p}\right)\right\}^{\frac{\alpha}{\alpha-p}}
$$




$$
+\frac{\beta-p}{p \beta\left(\beta a_{0}\right)^{\frac{p}{\beta-p}}}\left\{\int_{\mathbb{R}^{N}}\left(\left|\nabla \psi_{\delta}\right|^{p}+\left(A\left(\lambda^{-\frac{1}{p}} x\right)+V\left(\lambda^{-\frac{1}{p}} x\right)\right)\left|\psi_{\delta}\right|^{p}\right)\right\}^{\frac{\beta}{\beta-p}} .
$$

Since $A(0)=0, V(0)=0$ and $\left\|\nabla \phi_{\delta}\right\|_{p}^{p},\left\|\nabla \psi_{\delta}\right\|_{p}^{p}<\delta$, we know that there is $\Lambda_{\delta}>0$ such that for all $\lambda \geq \Lambda_{\delta}$, we have

$$
\max _{t \geq 0} I_{\lambda}\left(t \phi_{\delta}, t \psi_{\delta}\right) \leq\left(\frac{\alpha-p}{p \alpha\left(\alpha a_{0}\right)^{\frac{p}{\alpha-p}}}(5 \delta)^{\frac{\alpha}{\alpha-p}}+\frac{\beta-p}{p \beta\left(\beta a_{0}\right)^{\frac{p}{\beta-p}}}(5 \delta)^{\frac{\beta}{\beta-p}}\right) \lambda^{1-\frac{N}{p}} .
$$

Lemma 4.3 For any $\sigma>0$, there is $\Lambda_{\sigma}>0$ such that $\lambda \geq \Lambda_{\sigma}$, there is $\bar{w}_{\lambda} \in E$ with $\left\|\bar{w}_{\lambda}\right\|_{E}>$ $\rho_{\lambda}, I_{\lambda}\left(\bar{w}_{\lambda}\right) \leq 0$ and

$$
\max _{t \geq 0} I_{\lambda}\left(t \bar{w}_{\lambda}\right) \leq \sigma \lambda^{1-\frac{N}{p}}
$$

where $\rho_{\lambda}$ is defined in Lemma 4.1.

Proof This proof is similar to that of Lemma 4.3 in [7], so we omit the details.

\section{Proof of Theorem 2}

Proof By using Lemma 4.3, for any $\sigma>0$ with $0<\sigma<\alpha_{0}$, we choose $\Lambda_{\sigma}>0$ and define the mini-max level

$$
c_{\lambda}=\inf _{\gamma \in \Gamma_{\lambda}} \max _{t \in[0,1]} I_{\lambda}(\gamma(t)) \leq \sigma \lambda^{1-\frac{N}{p}} \quad \text { for all } \lambda \geq \Lambda_{\sigma}
$$

where $\Gamma_{\lambda}=\left\{\gamma \in C([0,1], E): \gamma(0)=0, \gamma(1)=\bar{w}_{\lambda}\right\}$.

By Proposition 3.1, we know that $I_{\lambda}$ satisfies the $(P S)_{c_{\lambda}}$ condition. Hence, by the mountain-pass theorem, there is $\left(u_{\lambda}, v_{\lambda}\right) \in E$ such that $I_{\lambda}\left(u_{\lambda}, v_{\lambda}\right)=c_{\lambda}$ and $I_{\lambda}^{\prime}\left(u_{\lambda}, v_{\lambda}\right)=0$. This shows $\left(u_{\lambda}, v_{\lambda}\right)$ is a weak solution of (2.1).

Moreover, note that $I_{\lambda}\left(u_{\lambda}, v_{\lambda}\right) \leq \sigma \lambda^{1-\frac{N}{p}}$ and $I_{\lambda}^{\prime}\left(u_{\lambda}, v_{\lambda}\right)=0$. Then

$$
\begin{aligned}
I_{\lambda}\left(u_{\lambda}, v_{\lambda}\right)= & I_{\lambda}\left(u_{\lambda}, v_{\lambda}\right)-\frac{1}{\theta} I_{\lambda}^{\prime}\left(u_{\lambda}, v_{\lambda}\right)\left(u_{\lambda}, v_{\lambda}\right) \\
= & \left(\frac{1}{p}-\frac{1}{\theta}\right)\left\|\left(u_{\lambda}, v_{\lambda}\right)\right\|_{E}^{p}+\left(\frac{1}{\theta}-\frac{1}{p^{*}}\right) \lambda \int_{\mathbb{R}^{N}} K(x)\left(\left|u_{\lambda}\right|^{p^{*}}+\left|v_{\lambda}\right|^{p^{*}}\right) \\
& +\lambda \int_{\mathbb{R}^{N}}\left(\frac{1}{\theta}\left(\left|u_{\lambda}\right|^{p} H_{s}\left(\left|u_{\lambda}\right|^{p},\left|v_{\lambda}\right|^{p}\right)+\left|v_{\lambda}\right|^{p} H_{t}\left(\left|u_{\lambda}\right|^{p},\left|v_{\lambda}\right|^{p}\right)\right)\right. \\
& \left.-\frac{1}{p} H\left(\left|u_{\lambda}\right|^{p},\left|v_{\lambda}\right|^{p}\right)\right) \\
\geq & \left(\frac{1}{p}-\frac{1}{\theta}\right)\left\|\left(u_{\lambda}, v_{\lambda}\right)\right\|_{E}^{p} .
\end{aligned}
$$

Furthermore, together with the diamagnetic inequality, we prove that $\left(u_{\lambda}, v_{\lambda}\right)$ satisfies the estimate (2.2). The proof is complete. 
Authors' contributions

The authors contributed equally in this article. All authors read and approved the final manuscript.

\section{Acknowledgements}

The authors would like to appreciate the referees for their precious comments and suggestions about the original manuscript. This research is supported by the National Natural Science Foundation of China (11271364) and the Fundamental Research Funds for the Central Universities (2012QNA46).

\section{Received: 5 October 2012 Accepted: 9 January 2013 Published: 24 January 2013}

\section{References}

1. Ambrosetti, A, Rabinowitz, PH: Dual variational methods in critical point theory and applications. J. Funct. Anal. 14, 349-381 (1973)

2. Benci, V: On critical point theory of indefinite functions in the presence of symmetries. Trans. Am. Math. Soc. 274, 533-572 (1982)

3. Brézis, H, Nirenberg, L: Positive solutions of nonlinear elliptic equation involving critical Sobolev exponents. Commun. Pure Appl. Math. 16, 437-477 (1983)

4. Cingolani, S, Nolasco, M: Multi-peaks periodic semiclassical states for a class of nonlinear Schrödinger equation. Proc. R. Soc. Edinb. 128, 1249-1260 (1998)

5. Del Pino, M, Felmer, PL: Semi-classical states for nonlinear Schrödinger equations. J. Funct. Anal. 149, 245-265 (1997)

6. Del Pino, M, Felmer, PL: Multi-peak bound states for nonlinear Schrödinger equations. Ann. Inst. Henri Poincaré, Anal. Non Linéaire 15, 127-149 (1998)

7. Ding, YH, Lin, FH: Solutions of perturbed Schrödinger equations with critical nonlinearity. Calc. Var. 30, 231-249 (2007)

8. Guedda, M, Veron, L: Quasilinear elliptic equations involving critical Sobolev exponents. Nonlinear Anal. 12, 879-902 (1989)

9. Jeanjean, L, Tanaka, K: Singularly perturbed elliptic problems with superlinear or asymptotically linear nonlinearities. Calc. Var. Partial Differ. Equ. 21, 287-318 (2004)

10. Kang, X, Wei, JC: On interacting bumps of semi-classical states of nonlinear Schrödinger equations. Adv. Differ. Equ. 5 899-928 (2000)

11. Li, YY: On a singularly perturbed elliptic equation. Adv. Differ. Equ. 2, 955-980 (1997)

12. Oh, YG: On positive multi-lump bound states of nonlinear Schrödinger equations under multiple well potential. Commun. Math. Phys. 131, 223-253 (1990)

13. Oh, YG: Existence of semiclassical bound states of nonlinear Schrödinger equations with potentials of the class $(V)$. Commun. Partial Differ. Equ. 13, 1499-1519 (1988)

14. Pistoia, A: Multi-peak solutions for a class of some results on a class of nonlinear Schrödinger equations. Nonlinear Differ. Equ. Appl. 9, 69-91 (2002)

15. Floer, A, Weinstein, A: Nonspreading wave packets for the cubic Schrödinger equation with a bounded potential. J. Funct. Anal. 69, 397-408 (1986)

16. Esteban, M, Lions, PL: Stationary solutions of nonlinear Schrödinger equation with an external magnetic field. In: Partial Differential Equations and the Calculus of Variations, Essays in Honor of E. De Giorgi, pp. 369-408. Brikhäuser, Basel (1990)

17. Arioli, G, Szulkin, A: A semilinear Schrödinger equation in the presence of a magnetic field. Arch. Ration. Mech. Anal. 170, 277-295 (2003)

18. Bartsch, T, Dancer, EN, Peng, S: On multi-bump semi-classical bound states of nonlinear Schrödinger equations with electromagnetic fields. Adv. Differ. Equ. 11, 781-812 (2006)

19. Cao, D, Tang, Z: Existence and uniqueness of multi-bump bound states of nonlinear Schrödinger equations with electromagnetic fields. J. Differ. Equ. 222, 381-424 (2006)

20. Cingolani, S: Semiclassical stationary states of nonlinear Schrödinger equation with an external magnetic field. J. Differ. Equ. 188, 52-79 (2003)

21. Han, P: Solutions for singular critical growth Schrödinger equation with magnetic field. Port. Math. 63, 37-45 (2006)

22. Kurata, K: Existence and semi-classical limit of the least energy solution to a nonlinear Schrödinger equation with electromagnetic fields. Nonlinear Anal. 41, 763-778 (2000)

23. Alves, CO, Ding, YH: Multiplicity of positive solutions to a $p$-Laplacian equation involving critical nonlinearity. J. Math. Anal. Appl. 279, 508-521 (2003)

24. Gloss, E: Existence and concentration of bound states for a $p$-Laplacian equation in $\mathbb{R}^{N}$. Adv. Nonlinear Stud. 10 , 273-296 (2010)

25. Liu, CG, Zheng, YQ: Existence of nontrivial solutions for $p$-Laplacian equations in $\mathbb{R}^{N}$. J. Math. Anal. Appl. 380, 669-679 (2011)

26. Manásevich, R, Mawhin, J: Boundary value problems for nonlinear perturbations of vector $p$-Laplacian-like operators. J. Korean Math. Soc. 5, 665-685 (2000)

27. Zhang, HX, Liu, WB: Existence of nontrivial solutions to perturbed $p$-Laplacian system in $\mathbb{R}^{N}$ involving critical nonlinearity. Bound. Value Probl. 2012, 53 (2012)

28. Dinu, TL: Entire solutions of Schrödinger systems with discontinuous nonlinearity and sign-changing potential. Math Model. Anal. 13(3), 229-242 (2006)

29. Dinu, TL: Entire solutions of multivalued nonlinear Schrödinger equations in Sobolev space with variable exponent. Nonlinear Anal. 65(7), 1414-1424 (2006)

30. Gazzola, F, Radulescu, V: A nonsmooth critical point theory approach to some nonlinear elliptic equations in unbounded domains. Differ. Integral Equ. 13, 47-60 (2000)

31. Ghanmi, A, Maagli, H, Radulescu, V, Zeddini, N: Large and bounded solutions for a class of nonlinear Schrödinger stationary systems. Anal. Appl. 7, 391-404 (2009)

32. Rabinowitz, PH: On a class of nonlinear Schrödinger equations. Z. Angew. Math. Phys. 43, 270-291 (1992)

33. Mawhin, J, Willem, M: Critical Point Theory and Hamiltonian Systems. Springer, New York (1989) 
34. Ghoussoub, N, Yuan, C: Multiple solutions for quasi-linear PDES involving the critical Sobolev and Hardy exponents. Trans. Am. Math. Soc. 352, 5703-5743 (2000)

35. Li, YY, Guo, QQ, Niu, PC: Global compactness results for quasilinear elliptic problems with combined critical Sobolev-Hardy terms. Nonlinear Anal. 74, 1445-1464 (2011)

36. Brézis, H, Lieb, E: A relation between pointwise convergence of functions and convergence of functional. Proc. Am. Math. Soc. 88, 486-490 (1983)

37. Pucci, P, Radulescu, V: The impact of the mountain pass theory in nonlinear analysis: a mathematical survey. Boll. Unione Mat. Ital., Ser. IX 3, 543-584 (2010)

38. Radulescu, V: Qualitative Analysis of Nonlinear Elliptic Partial Differential Equations: Monotonicity, Analytic and Variational Methods. Contemporary Mathematics and Its Applications. Hindawi Publ. Corp, New York (2008)

doi:10.1186/1687-2770-2013-11

Cite this article as: Zhang et al.: Existence of nontrivial solutions for perturbed $p$-Laplacian system involving critical nonlinearity and magnetic fields. Boundary Value Problems 2013 2013:11.

Submit your manuscript to a SpringerOpen ${ }^{\circ}$ journal and benefit from:

- Convenient online submission

Rigorous peer review

- Immediate publication on acceptance

- Open access: articles freely available online

- High visibility within the field

- Retaining the copyright to your article

Submit your next manuscript at $>$ springeropen.com 